INJE-TP-98-3/hep-th9803227

\title{
Probing the BTZ black hole with test fields
}

\author{
H.W. Lee, N.J. Kim and Y. S. Myung \\ Department of Physics, Inje University, Kimhae 621-749, Korea
}

\begin{abstract}
We introduce a set of scalar fields as test fields to study the dynamical behaviors of the BTZ(Banados-Teitelboim-Zanelli) black hole. These include minimally coupled scalar, conformally coupled scalar, dilaton, and tachyon. To calculate the decay rate of the BTZ black hole, we consider both the Dirichlet boundary condition at spatial infinity and the stability condition. It turns out that the tachyon may be a relevant field to get information of the BTZ black hole.
\end{abstract}


Recently anti-de Sitter spacetime(AdS) has attracted much interest. It appears that the conjecture relating the string theory on AdS to conformal field theory $(\mathrm{CFT})$ on the boundary may resolve many problems in black hole physics [囬]. Sfetsos and Skenderis showed that 4d black hole(5d black hole) correspond to U-dual to $\mathrm{BTZ} \times S^{2}\left(\mathrm{BTZ} \times S^{3}\right)$ [2]. They calculated the entropies of non-extremal $4 \mathrm{~d}$ and $5 \mathrm{~d}$ black holes by applying Carlip's approach to the BTZ black hole. The BTZ black hole(locally, (2+1)-dimensional anti-de Sitter spacetime : $\mathrm{AdS}_{3}$ ) has no curvature singularity [3] and is considered as a prototype for the general CFT/AdS correspondence [1]. This is actually an exact solution of string theory [4] and there is an exact CFT with it on the boundary. Carlip has shown that the physical boundary degrees of freedom account for the Bekenstein-Hawking entropy of the BTZ black hole correctly [5].

In this letter we investigate the dynamical behavior of the BTZ black hole with test scalar fields. Apart from counting the microstates of black holes, the dynamical behavior is also an important issue [6,7]. This is so because the greybody factor(absorption cross-section) for the black hole arises as a consequence of scattering of test field off the gravitational potential barrier surrounding the horizon. That is, this is an effect of spacetime curvature. Together with the Bekenstein-Hawking entropy, this seems to be the strong hint of a deep and mysterious connection between curvature and statistical mechanics. Birmingham et al. showed that the greybody factor for the BTZ black hole has the same form as the one for $5 \mathrm{~d}$ black hole in the dilute gas approximation [8]. However a minimally coupled scalar was used for this end and the boundary condition at spatial infinity is not required for calculation. It is found that the dilaton(fixed scalar) plays an important role in probing the dynamical behaviors, when one does not impose any boundary condition at the infinity [9]. Also a conformally coupled scalar may be used for studying the BTZ black hole [10]. But it turns out that this is not appropriate for describing the particle creation by the BTZ black hole [11. As a result, it is very important to inquire which one is suitable for studying the dynamical behavior of the BTZ black hole among a set of scalar fields.

We start with the BTZ black hole spacetime as 


$$
\bar{g}_{\mu \nu}=\left(\begin{array}{ccc}
-\left(M-r^{2} / l^{2}\right) & -J / 2 & 0 \\
-J / 2 & r^{2} & 0 \\
0 & 0 & f^{-2}
\end{array}\right)
$$

with $f^{2}=r^{2} / l^{2}-M+J^{2} / 4 r^{2}$. The metric $\bar{g}_{\mu \nu}$ is singular at $r=r_{ \pm}$,

$$
r_{ \pm}=\frac{M l^{2}}{2}\left\{1 \pm\left[1-\left(\frac{J}{M l}\right)^{2}\right]^{1 / 2}\right\}
$$

with $M=\left(r_{+}^{2}+r_{-}^{2}\right) / l^{2}, J=2 r_{+} r_{-} / l$. Here $r_{+}\left(r_{-}\right)$correspond to the outer(inner) horizon. For convenience, we list the Hawking temperature $T_{H}$, the area of horizon $\mathcal{A}_{H}$, and the angular velocity at the horizon $\Omega_{H}$ as

$$
T_{H}=\left(r_{+}^{2}-r_{-}^{2}\right) / 2 \pi l^{2} r_{+}, \quad \mathcal{A}_{H}=2 \pi r_{+}, \quad \Omega_{H}=J / 2 r_{+}^{2} .
$$

To study the propagation specifically, we introduce the small test field $(\Psi)$ around the background solution (1). In three dimensions we have no propagating degrees of freedom for graviton $h_{\mu \nu}$ [9] and vector fields are dual to scalar fields [12]. Also the two-form field $B_{\mu \nu}$ has no physical degrees of freedom for $d=3$. Hence the physical degrees of freedom in the BTZ black hole turn out to be a set of scalar fields which satisfy

$$
\bar{\nabla}^{2} \Psi+\frac{s}{l^{2}} \Psi=0
$$

where $s$ is a new coupling constant. $s=0,3 / 4,-8$ cases correspond to minimally coupled scalar, conformally coupled scalar, and dilaton, respectively. Considering the $t$ and $x$-translational symmetries of the background spacetime (1), we can decompose $\Psi$ into frequency modes in these variables [13] as

$$
\Psi(t, x, r)=e^{-i \omega t} e^{i \mu x} \tilde{\Psi}(r), \mu \in Z
$$

Together (4) with (11) leads to

$$
\left[f^{2} \partial_{r}^{2}+\left\{\frac{1}{r}\left(\partial_{r} r f^{2}\right)\right\} \partial_{r}-\frac{J \mu \omega}{r^{2} f^{2}}+\frac{\omega^{2}}{f^{2}}+\frac{M-\frac{r^{2}}{l^{2}}}{r^{2} f^{2}} \mu^{2}\right] \tilde{\Psi}+\frac{s}{l^{2}} \tilde{\Psi}=0 .
$$


Let us calculate the absorption cross-section to study the dynamical behavior of the BTZ black hole. First we note that the spatial infinity of the BTZ black hole is timelike(AdS 3 is not globally hyperbolic), so that information may enter or exit from the boundary at infinity. Thus one impose the boundary condition at infinity to obtain the sensible results [10]. Here we require the Dirichlet condition as

$$
\lim _{r \rightarrow \infty} \sqrt{r} \tilde{\Psi}_{\infty}=0
$$

Second, since it is hard to find a solution to (6) directly, we use a matching procedure. The spacetime is divided into two regions: the near region $\left(r \sim r_{+}\right)$and far region $(r \rightarrow \infty)$ [6,7]. We now study each region in turn. For the far region $(r \rightarrow \infty)$, (6) becomes

$$
\tilde{\Psi}_{\infty}^{\prime \prime}+\frac{3}{r} \tilde{\Psi}_{\infty}^{\prime}+\frac{s}{r^{2}} \tilde{\Psi}_{\infty}=0
$$

Here one finds the far region solution

$$
\tilde{\Psi}_{\mathrm{far}}(r)=\frac{1}{r}\left(A_{1} r^{-\sqrt{1-s}}+A_{2} r^{+\sqrt{1-s}}\right)
$$

In order to obtain the near region behavior, we introduce the variable $z=\frac{r^{2}-r_{+}^{2}}{r^{2}-r_{-}^{2}}, \quad 0 \leq$ $z \leq 1$. Then (6) becomes

$$
z(1-z) \frac{d^{2} \tilde{\Psi}}{d z^{2}}+(1-z) \frac{d \tilde{\Psi}}{d z}+\left(\frac{A_{1}}{z}+\frac{s / 4}{1-z}+B_{1}\right) \tilde{\Psi}=0
$$

where $A_{1}=\left(\frac{\omega-\mu \Omega_{H}}{4 \pi T_{H}}\right)^{2}, B_{1}=-\frac{r_{-}^{2}}{r_{+}^{2}}\left(\frac{\omega-\mu \Omega_{H} r_{+}^{2} / r_{-}^{2}}{4 \pi T_{H}}\right)^{2}$. The solution for (10) is given by the hypergeometric functions

$$
\begin{aligned}
\tilde{\Psi}_{\text {near }}(z)=C_{1} z^{-i \sqrt{A_{1}}}(1-z)^{(1-\sqrt{1-s}) / 2} F(a, b, c ; z) \\
\quad+C_{2} z^{i \sqrt{A_{1}}}(1-z)^{(1-\sqrt{1-s}) / 2} F(b-c+1, a-c+1,2-c ; z),
\end{aligned}
$$

where

$$
\begin{aligned}
& a=\sqrt{B_{1}}-i \sqrt{A_{1}}+(1-\sqrt{1-s}) / 2, \\
& b=-\sqrt{B_{1}}-i \sqrt{A_{1}}+(1-\sqrt{1-s}) / 2, \\
& c=1-2 i \sqrt{A_{1}} .
\end{aligned}
$$


and $C_{1}$ and $C_{2}$ are to-be-determined constants. At the near horizon $\left(r \sim r_{+}, z \sim 0\right)$, (11) becomes

$$
\begin{aligned}
\tilde{\Psi}_{\text {near }}(0) & \simeq C_{1} z^{-i \sqrt{A_{1}}}+C_{2} z^{i \sqrt{A_{1}}} \\
& =C_{1}\left(\frac{2 r_{+}}{r_{+}^{2}-r_{-}^{2}}\right)^{-i \sqrt{A_{1}}} e^{-i \sqrt{A_{1}} \ln \left(r-r_{+}\right)}+C_{2}\left(\frac{2 r_{+}}{r_{+}^{2}-r_{-}^{2}}\right)^{i \sqrt{A_{1}}} e^{i \sqrt{A_{1}} \ln \left(r-r_{+}\right)} .
\end{aligned}
$$

Considering an ingoing mode at horizon, we have $C_{2}=0$. Hence the near region solution is

$$
\tilde{\Psi}_{\text {near }}(z)=C_{1} z^{-i \sqrt{A_{1}}}(1-z)^{(1-\sqrt{1-s}) / 2} F(a, b, c ; z) .
$$

To use matching procedure we need to know the behaviour of (14) near $z=1$. This can be obtained by the transformation rule $(z \rightarrow 1-z)$ for hypergeometric function [14]. Using this rule we can obtain

$$
\tilde{\Psi}_{n \rightarrow f}(z)=\frac{1}{r}\left(C_{1} E_{1} r^{-\sqrt{1-s}}+C_{1} E_{2} r^{+\sqrt{1-s}}\right)
$$

where

$$
\begin{aligned}
& E_{1}=\frac{\Gamma\left(1-2 i \sqrt{A_{1}}\right) \Gamma(-\sqrt{1-s})\left(r_{+}^{2}-r_{-}^{2}\right)^{(1+\sqrt{1-s}) / 2}}{\left.\Gamma\left(\frac{1}{2}+\sqrt{B_{1}}-i \sqrt{A_{1}}-\frac{\sqrt{1-s}}{2}\right) \Gamma\left(\frac{1}{2}-\sqrt{B_{1}}-i \sqrt{A_{1}}-\frac{\sqrt{1-s}}{2}\right)\right)}, \\
& E_{2}=\frac{\Gamma\left(1-2 i \sqrt{A_{1}}\right) \Gamma(\sqrt{1-s})\left(r_{+}^{2}-r_{-}^{2}\right)^{(1-\sqrt{1-s}) / 2}}{\left.\left.\Gamma\left(\frac{1}{2}-\sqrt{B_{1}}-i \sqrt{A_{1}}+\frac{\sqrt{1-s}}{2}\right)\right) \Gamma\left(\frac{1}{2}+\sqrt{B_{1}}-i \sqrt{A_{1}}+\frac{\sqrt{1-s}}{2}\right)\right)} .
\end{aligned}
$$

In matching (9) with (15), one needs to classify the next steps according to the values of $s$.

(i) $s>1$ case

In this case, (9) becomes

$$
\tilde{\Psi}_{\mathrm{far}}(r)=\frac{1}{r}\left(A_{\mathrm{in}} e^{-i \sqrt{s-1} \ln r}+A_{\mathrm{out}} e^{+i \sqrt{s-1} \ln r}\right)
$$

with two unknown coefficients $A_{\text {in }}$ and $A_{\text {out }}$. The first(second) term correspond to ingoing(outgoing) waves at the far region. This can be confirmed by calculating the corresponding flux as $\mathcal{F}_{\text {in }}=-4 \pi \sqrt{s-1}\left|A_{\text {in }}\right|^{2}\left(\mathcal{F}_{\text {out }}=4 \pi \sqrt{s-1}\left|A_{\text {out }}\right|^{2}\right)$. Comparing this with $(15)$ leads to $A_{\text {in }}=C_{1} E_{1}$ and $A_{\text {out }}=C_{1} E_{2}$. The absorption coefficient is calculated as 


$$
\mathcal{A}=1-\left|\frac{A_{\text {out }}}{A_{\text {in }}}\right|^{2}=\frac{\left(e^{2 \sqrt{s-1} \pi}-1\right)\left(e^{\omega^{\prime} / T_{H}}-1\right)}{\left(e^{\omega_{R}^{\prime} / 2 T_{R}+\sqrt{s-1} \pi}+1\right)\left(e^{\omega_{L}^{\prime} / 2 T_{L}+\sqrt{s-1} \pi}+1\right)},
$$

where $\omega^{\prime}=\omega-\mu \Omega_{H}, \omega_{L / R}^{\prime}=\omega \mp \mu \Omega_{H} r_{+} / r_{-}$and left/right temperatures are defined by

$$
\frac{1}{T_{L / R}}=\frac{1}{T_{H}}\left(1 \pm \frac{r_{-}}{r_{+}}\right)
$$

The absorption corss-section is given by $\sigma_{\mathrm{abs}}=\mathcal{A} / \omega$ in three dimensions. Finally the decay rate is given as

$$
\Gamma_{\text {fixed }}=\frac{\sigma_{\text {abs }}}{e^{\frac{\omega^{\prime}}{T_{H}}}-1}=\frac{1}{\omega} \frac{e^{2 \sqrt{s-1} \pi}-1}{\left(e^{\omega_{R}^{\prime} / 2 T_{R}+\sqrt{s-1} \pi}+1\right)\left(e^{\omega_{L}^{\prime} / 2 T_{L}+\sqrt{s-1} \pi}+1\right)} .
$$

(ii) $s=1$ case

Here the far region solution is

$$
\tilde{\Psi}_{\text {far }}(r)=\frac{1}{r}\left(A_{1}+A_{2} \ln r\right) .
$$

For our purpose this can be rearragned to give

$$
\tilde{\Psi}_{\mathrm{far}}(r)=\frac{A_{\text {in }}}{r}(1-i \ln r)+\frac{A_{\mathrm{out}}}{r}(1+i \ln r) .
$$

The first term give us the ingoing flux $\left(\mathcal{F}_{\text {in }}=-4 \pi\left|A_{\text {in }}\right|^{2}\right)$ and the second is outgoing flux $\left(\mathcal{F}_{\text {out }}=-4 \pi\left|A_{\text {out }}\right|^{2}\right)$. We note that the transformation rule in deriving (15) is valid only for $c \neq a+b \pm m$ with integer $m$. Considering (12) with $s=1$, we have $c=a+b$. In this case we should use a different rule for $z \rightarrow 1-z[14$. Using this we obtain

$$
\tilde{\Psi}_{n \rightarrow f}(z)=\frac{1}{r}\left(C_{1} D_{1}-C_{1} D_{2} \ln r\right)
$$

where

$$
\begin{aligned}
D_{1} & =\frac{\Gamma\left(1-2 i \sqrt{A_{1}}\right)\left(r_{+}^{2}-r_{-}^{2}\right)^{1 / 2}}{\Gamma\left(\frac{1}{2}+\sqrt{B_{1}}-i \sqrt{A_{1}}\right) \Gamma\left(\frac{1}{2}-\sqrt{B_{1}}-i \sqrt{A_{1}}\right)}\left[2 \psi(1)-\psi(a)-\psi(b)-\ln \left(r_{+}^{2}-r_{-}^{2}\right)\right] \\
D_{2} & =-2 \frac{\Gamma\left(1-2 i \sqrt{A_{1}}\right)\left(r_{+}^{2}-r_{-}^{2}\right)^{1 / 2}}{\Gamma\left(\frac{1}{2}+\sqrt{B_{1}}-i \sqrt{A_{1}}\right) \Gamma\left(\frac{1}{2}-\sqrt{B_{1}}-i \sqrt{A_{1}}\right)}
\end{aligned}
$$

Here $\psi$ is the digamma function. Comparing (23) and (24), one finds $A_{\text {in }}=C_{1}\left(D_{1}-i D_{2}\right) / 2$ and $A_{\text {out }}=C_{1}\left(D_{1}+i D_{2}\right) / 2$. The absorption coefficient is calculated as 


$$
\mathcal{A}=1-\left|\frac{A_{\text {out }}}{A_{\text {in }}}\right|^{2}=\frac{8 \Im\left[2 \psi(1)-\psi(a)-\psi(b)-\ln \Delta_{-}\right]}{\left|2 \psi(1)-\psi(a)-\psi(b)-\ln \Delta_{-}-2 i\right|^{2}}
$$

with $\Delta_{-}=\left(r_{+}^{2}-r_{-}^{2}\right)$. Here $\Im[\cdots]$ means the imaginary part of its argument. The tachyon with $s>0$ may come from the low energy string effective action [15]. This may be adjusted to satisfy (4) with $0<s \leq 2$, when one chooses a suitable convention for the BTZ black hole [16. In this sense the tachyon may play a role in deriving information for the BTZ black hole.

(iii) $\frac{3}{4}<s<1$ case

In this regime, (9) takes the form

$$
\tilde{\Psi}_{\text {far }}(r)=\frac{A_{\text {in }}}{r}\left(r^{\sqrt{1-s}}+i r^{-\sqrt{1-s}}\right)+\frac{A_{\text {out }}}{r}\left(r^{\sqrt{1-s}}-i r^{-\sqrt{1-s}}\right) .
$$

The first(second) term correspond to ingoing flux $=-8 \pi \sqrt{1-s}\left|A_{\text {in }}\right|^{2}$ (outgoing flux $=$ $\left.8 \pi \sqrt{1-s}\left|A_{\text {out }}\right|^{2}\right)$. From (28) and (13) we find $A_{\text {in }}=-i C_{1}\left(E_{1}+i E_{2}\right) / 2 A_{\text {out }}=i C_{1}\left(E_{1}-\right.$ $\left.i E_{2}\right) / 2$. The absorption coefficient is

$$
\mathcal{A}=1-\left|\frac{A_{\text {out }}}{A_{\text {in }}}\right|^{2}=1-\frac{\left|E_{1}-i E_{2}\right|^{2}}{\left|E_{1}+i E_{2}\right|^{2}}
$$

where $E_{1}$ and $E_{2}$ are given by (16) and (17). In the extremal limit $\left(r_{+}=r_{-}\right)$one finds the total reflection $(\mathcal{A}=0)$.

(iv) $0<s \leq \frac{3}{4}$ case

Equation (9) can be rewritten as

$$
\tilde{\Psi}_{\text {far }}(r)=\frac{1}{r}\left[\left(A_{\text {in }}+A_{\text {out }}\right) e^{\sqrt{1-s}}+i\left(A_{\text {in }}-A_{\text {out }}\right) e^{-\sqrt{1-s}}\right] .
$$

If one requires the boundary condition, then one finds $A_{\text {in }}=-A_{\text {out }}$. In this case the total flux $\left(\mathcal{F}_{\text {in }}+\mathcal{F}_{\text {out }}\right)$ is zero, and so one finds the total reflection. Further (30) with $A_{\text {in }}=-A_{\text {out }}$ cannot be matched with (15). This means that the field with $s \leq 3 / 4$ is not useful for probing the dynamical behaviour of BTZ black hole. We note that a conformally coupled scalar takes the value $s=3 / 4$ [10]. As a result, this field seems to be not appropriate for studying the particle creation by the BTZ black hole. Also Kim et al. pointed out that the 
conformally coupled scalar is not useful for investigating the Hawking radiation of the BTZ black hole. They showed that if one uses a conformally coupled matter in the conformallyflat spacetime, there is no particle creation in the BTZ black hole. This supports that our result for a conformally coupled scalar is correct.

(v) $s=0$ case

In this case, (9) takes the form

$$
\tilde{\Psi}_{\text {far }}(r)=A_{1}+\frac{A_{2}}{r^{2}}
$$

Using the transformation rule for $c=a+b+1$ [14], we obtain the asymptotic form

$$
\tilde{\Psi}_{n \rightarrow f}(z) \simeq C_{1} F_{1}+\frac{C_{1} F_{2}}{r^{2}}-\frac{C_{1} F_{3} \ln r}{r^{2}}
$$

where

$$
\begin{aligned}
& F_{1}=\frac{\Gamma(a+b+1)}{\Gamma(a+1) \Gamma(b+1)}, \\
& F_{2}=\Delta_{-}\left[\ln \Delta_{-}+\psi(a+1)+\psi(b+1)-\psi(1)-\psi(2)\right] a b F_{1} \\
& F_{3}=\Delta_{-} a b F_{1} .
\end{aligned}
$$

Here $a b=-l^{2}\left(\omega^{2}-\mu^{2}\right) / 2 \Delta_{-}$. If $\mu=0$ and $\omega \ll 1 / l, F_{2}$ and $F_{3}$ is small compared to $F_{1}$. Thus we find $A_{1}=C_{1} F_{1}$ and $A_{2} \simeq 0$. However this does not satisfy the Dirichlet boundary condition unless $A_{1}=C_{1} F_{1}=0$. That is, we have no information for the BTZ black hole with a minimally coupled $\operatorname{scalar}(s=0)$. Birmingham et al. calculated the decay rate of the BTZ black hole with this scalar [8],

$$
\Gamma_{\min }=\frac{\sigma_{\mathrm{abs}}^{\min }}{e^{\frac{\omega}{T_{H}}}-1}=\frac{\pi^{2} l^{2} \omega}{\left(e^{\frac{\omega}{2 T_{R}}}-1\right)\left(e^{\frac{\omega}{2 T_{L}}}-1\right)} .
$$

However they did not ask the boundary condition at spatial infinity. If one requires (7) it is hard to calculate the decay rate for the BTZ black hole.

(vi) $s<0$ case

Here, (9) can be rewritten as 


$$
\tilde{\Psi}_{\text {far }}(r)=\frac{1}{r}\left[\left(A_{\text {in }}+A_{\text {out }}\right) r^{\sqrt{1-s}}+i\left(A_{\text {in }}-A_{\text {out }}\right) r^{-\sqrt{1-s}}\right] .
$$

Considering the boundary condition, then one finds $A_{\text {in }}=-A_{\text {out }}$. In this case the total flux $\left(\mathcal{F}_{\text {in }}+\mathcal{F}_{\text {out }}\right)$ is zero, and this means the total reflection. Further (35) with $A_{\text {in }}=-A_{\text {out }}$ cannot be matched with (15). This implies that the field with $s<0$ may not be useful for probing the dynamical behaviour of BTZ black hole. The dilaton and massive scalar field which satisfies $\nabla^{2} \Psi-m^{2} \Psi=0$, belong to this case 9, 17.

In conclusion, we introduce a set of scalar fields to study the Hawking radiation of the BTZ black hole. These are minimally coupled scalar, conformally coupled scalar, and dilaton including tachyon and massive scalar field. If one does not require the boundary condition at spatial infinity, the dilaton can be regarded as the best field to get information about the BTZ black hole. As was shown in the $s<0$ case, it turns out that the dilaton with $s=-8$ is not appropriate for probing the BTZ black hole. However, the tachyon may be emerged as a candidate for inquiring the BTZ black hole. The tachyon is defined as a field with $m^{2}<0$ and thus causes the difficulty in the Minkowski spacetime. On the other hand, the tachyon may play an important role in the anti-de Sitter spacetime. Because of the boundary condition at spatial infinity, the kinetic energy of a scalar field in $\mathrm{AdS}_{3}$ cannot vanish. Thus the stability of the BTZ black hole requires not that $m^{2}$ should be positive $(s<0)$ but $m^{2}$ should be no smaller than a certain negative lower bound [18. For d-dimensional AdS case, this is approximately given by $m^{2} \geq-(d-1)^{2} / 4$. Then test fields should satisfy the stability condition : $m^{2} \geq-1(s \leq 1)$.

Hence if we take both the stability $(s \leq 1)$ of the BTZ black hole and the boundary condition at spatial infinity $(s>3 / 4)$ seriously, the relevant field will be the tachyon with $3 / 4<s \leq 1$. Also the dilaton with $s=-8$ may be excluded from our consideration. In this sense, the remaining fields (minimally coupled $\operatorname{scalar}(s=0)$, conformally coupled $\operatorname{scalar}(s=3 / 4)$, and massive scalar field $(s<0))$ are not suitable for our purposes. 


\section{ACKNOWLEDGEMENT}

This work was supported in part by the Basic Science Research Institute Program, Minstry of Education, Project NOs. BSRI-97-2413 and BSRI-97-2441. 


\section{REFERENCES}

[1] J. Maldacena, hep-th/9711200; G.T. Horowitz and H. Oguri, hep-th/9802116; E. Witten, hep-th/9803131; G.T. Horowitz and S. Ross, hep-th/9803085.

[2] K. Sfetsos and K. Skenderis, hep-th/9711138.

[3] M. Banados, C. Teitelboim and A. Zanelli, Phys. Rev. Lett. 69 (1992)1849; S. Carlip, Class. Quant. Grav. 12 (1995)2853.

[4] G. Horowitz and D. Welch, Phys. Rev. Lett. 71 (1993)328; N. Kaloper, Phys. Rev. D48 (1993)2598; A. Ali and A. Kumar, Mod. Phys. Lett. A8 (1993)2045.

[5] S. Carlip, Phys. Rev. D51 (1995)632; D55 (1997)878.

[6] A. Dhar, G. Mandal and S. Wadia, Phys. Lett. B388 (1996)51; S. Das, G. Gibbons and S. Mathur, Phys. Rev. Lett. 78 (1997)417.

[7] C. Callan, S. Gubser, I.G. Klebanov and A. Tseytlin, Nucl. Phys. B489 (1997)65; M. Krasnitz and I.G. Klebanov, hep-th/9703216; B. Kol and A. Rajaraman, Phys. Rev. D56 (1997)983; H.W. Lee, Y.S. Myung and J.Y. Kim, Phys. Lett. B410 (1997)6; hepth/9708099.

[8] D.B. Birmingham, I. Sachs and S. Sen, Phys. Lett. B413 (1997)281.

[9] H.W. Lee, N.J. Kim, and Y.S. Myung, hep-th/9803080.

[10] G. Lifschytz and M. Oritz, Phys. Rev. D49 (1994)1929; S. Hyun, G.H. Lee and J.H. Yee, Phys. Lett. B322 (1994)182; J. Chan and R. Mann, gr-qc/9612026.

[11] H. Kim, J. Oh and C. Ahn, hep-th/9708072.

[12] I. Bakas, M. Bourdeau and G. Cardoso, hep-th/9706032.

[13] R. Gregory and R. Laflamme, Phys. Rev. Lett. 70 (1993)2837; H.W. Lee, N.J. Kim, Y.S. Myung and J.Y. Kim, hep-th/9801152. 
[14] M. Abramowitz and I. Stegun, Handbook of Mathematical Functions (Academic Press, New York, 1966) p.559.

[15] T. Banks, Nucl. Phys. B361 (1991)166; D. McGuigan, C. Nappi, and S. Yost, Nucl. Phys. B375 (1992) 421; K. Sfetsos, Nucl. Phys. B389 (1993)424; R. Raiten, Nucl. Phys. B416 (1994)881; H.W. Lee, Y.S. Myung and J.Y. Kim, Phys. Rev. D52 (1995)2214.

[16] K. Ghoroku and A.L. Larsen, Phys. Lett. B328 (1994)28.

[17] I. Ichinose and K. Satoh, Nucl. Phys. B447 (1995)340.

[18] E. Witten, hep-th/9802150; B. Breitenlohner and D.Z. Freedman, Phys. Lett. B115 (1982) 197; Ann. Phys. 114 (1982) 117. 